REC FIVEO

JAN 261998

OgT

GA-A22684

CONF-971216-.

\title{
CONTROL OF PLASMA POLOIDAL SHAPE AND POSITION IN THE DIII-D TOKAMAK
}

by

M.L. WALKER, D.A. HUMPHREYS, and J.R. FERRON

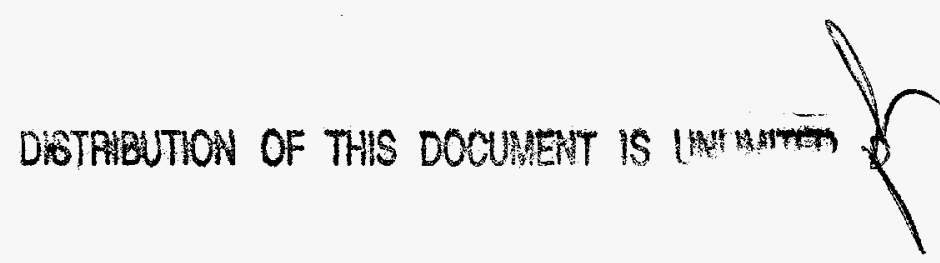

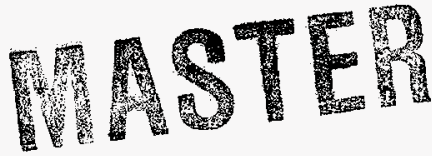

NOVEMBER 1997 


\section{DISCLAMER}

This report was prepared as an account of work sponsored by an agency of the United States Government. Neither the United States Government nor any agency thereof, nor any of their employees, makes any warranty, express or implied, or assumes any legal liability or responsibility for the accuracy, completeness, or usefulness of any information, apparatus, product, or process disclosed, or represents that its use would not infringe privately owned rights. Reference herein to any specific commercial product, process, or service by trade name, trademark, manufacturer, or otherwise does not necessarily constitute or imply its endorsement, recommendation, or favoring by the United States Government or any agency thereof. The views and opinions of authors expressed herein do not necessarily state or reflect those of the United States Government or any agency thereof. 


\section{DISCLAIMER}

Portions of this document may be illegible electronic image products. Images are produced from the best available original document. 


\title{
CONTROL OF PLASMA POLOIDAL SHAPE AND POSITION IN THE DIII-D TOKAMAK
}

\author{
by \\ M.L. WALKER, D.A. HUMPHREYS, and J.R. FERRON
}

This is a preprint of a paper presented at the $36^{\text {th }}$ IEEE Conference on Decision and Control, December 10-12, 1997, in San Diego, California and to be published in the Proceedings.

\author{
Work supported by \\ the U.S. Department of Energy under \\ Contract No. DE-AC03-89ER51114
}

GA PROJECT 3466

NOVEMBER 1997 


\title{
Control of Plasma Poloidal Shape and Position in the DIII-D Tokamak ${ }^{*}$
}

\author{
M.L.Walker, D.A.Humphreys, J.R.Ferron \\ General Atomics, P.O. Box 85608, San Diego, California 92816-9784 \\ e-mail:walker@gav.gat.com
}

\section{Introduction and Description of Problem}

Historically, tokamak control design has been a combination of theory driving an initial control design and empirical tuning of controllers to achieve satisfactory performance. This approach was in line with the focus of past experiments on simply obtaining sufficient control to study many of the basic physics issues of plasma behavior. However, in recent years existing experimental devices have required increasingly accurate control. New tokamaks such as ITER or the eventual fusion power plant must achieve and confine burning fusion plasmas, placing unprecedented demands on regulation of plasma shape and position, heat flux, and burn characteristics. Control designs for such tokamaks must also function well during initial device operation with minimal empirical optimization required. All of these design requirements imply a heavy reliance on plasma modeling and simulation. Thus, plasma control design has begun to use increasingly modern and sophisticated control design methods. This paper describes some of the history of plasma control for the DIII-D tokamak as well as the recent effort to implement modern controllers. This effort improves the control so that we may obtain better physics experiments and simultaneously develop the technology for designing controllers for next-generation tokamaks.

The DIII-D tokamak is toroidal device with a D-shaped cross-section having a major radius of $1.7 \mathrm{~m}$ and a characteristic time constant of several milliseconds for field penetration through the vacuum vessel. The time constant of the dominant instability is typically comparable to this field penetration time. Figure 1 shows a cross-section of DIII-D with lines of constant flux shown to illustrate the plasma. The primary purpose of the Ohmic heating coil (E-coil) solenoid is to induce current in the plasma through transformer action. This heats the plasma through resistive losses and provides a distributed current which can be shaped by external currents. The 18 poloidal field coils (F-coils) F1A to F9B are used to control the shape and position of the plasma. Shaping power supplies act as voltage sources in series with certain of the F-coils to provide the necessary shaping currents. Characteristics of the device which make for challenging control problems are the highly nonlinear nature of the plasma response and of the shaping power supplies, the high degree of coupling between the responses of the plasma to various F-coils, and the fact that the plasma vertical motion is highly unstable. An additional problem is that it's difficult to determine the plasma shape in real-time because the shape cannot be measured directly but must be reconstructed from

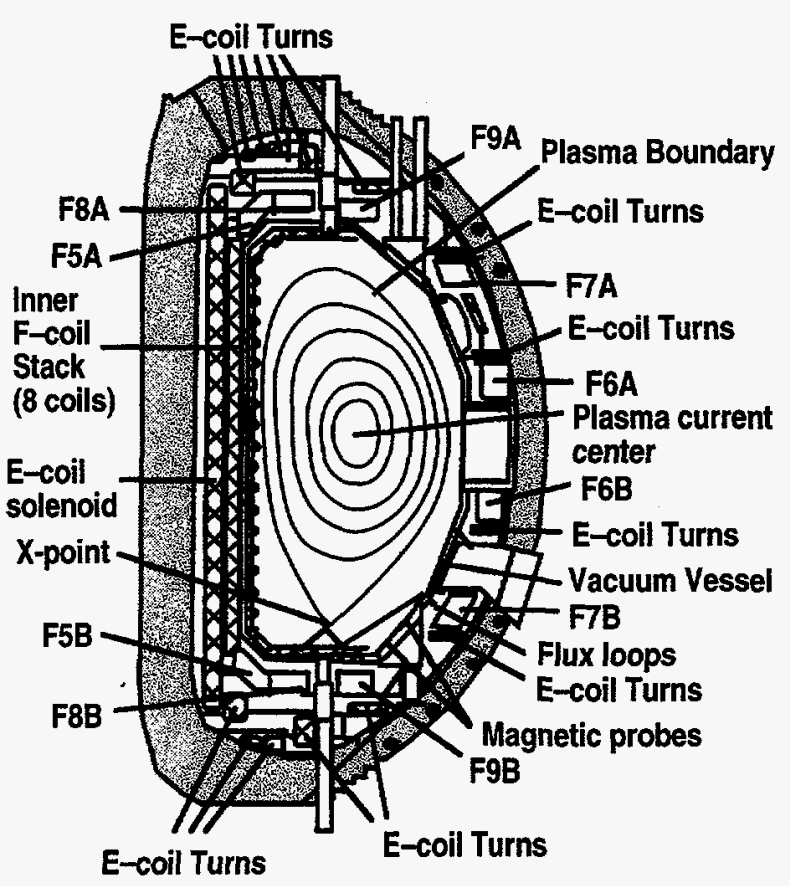

Fig. 1. Cross-section of DIII-D Tokamak (torus).

discrete magnetic field and flux measurements. Measurements which are used to estimate the shape and position are magnetic field at 29 magnetic probes on the inner side of the vacuum vessel ( 2 probes shown), fluxes at 41 flux loops on the outer side of the vessel and on the F-coils (1 loop shown), currents in $6 \mathrm{E}$ - and $18 \mathrm{~F}$-coils, total toroidal current in the plasma and containment vessel, and optical measurements of plasma internal magnetic field.

The problem to be considered here is the control of the shape and position of a plasma in DIII-D. The traditional approach to solving this control problem has been to decouple controls as much as possible. In the past this has often meant that the effect on a particular plasma parameter from coils other than the coil actually being used to control that parameter were ignored. More modern efforts at shape control seek to take these effects into account in the control design. Some controls may still be decoupled, however, to minimize the size and complexity of resulting controllers. For example, the response time needed for vertical position control is much faster than that needed for shaping or plasma current control, and is therefore handled by a seperate controller. Plasma current control also closes the loop seperately because the E-coil

\footnotetext{
*Work supported by the U.S. Department of Energy under Contract DE-AC03-89ER51114.
} 
produces approximately constant flux across the vessel interior and thus has little effect on the shape which is determined by relative values of flux within the interior. This decoupling of control is in contrast with proposed controllers for tokamaks such as ITER where shaping, plasma current, and vertical position control are integrated because of a much smaller coil set and more commensurate time scales.

In the following sections, we describe the evolution of plasma shape and position control at DIII-D with emphasis on recent work. This control consists of two equally challenging problems - the problem of identifying what the plasma actually looks like in real time, i.e. measuring the parameters to be controlled, and the task of determining the feedback algorithm which best controls these plasma parameters in a multiple input-output system. Recent implementation of a real time plasma equilibrium reconstruction algorithm seems to solve the longstanding problem of obtaining sufficiently accurate plasma shape and position estimation. Stabilization of the open-loop unstable vertical motion is also viewed as a solved problem. The primary remaining problem appears to be how best to command the power supplies to achieve a desired shaping control response. We will describe our effort to understand and apply linearized models of plasma evolution to development and implementation of multivariable plasma controllers.

\section{Vertical Position Control}

An analog controller for DIII-D plasma vertical position was originally developed based on a single-filament plasma model and then empirically optimized. An extensive analysis based on this model is presented in [1]. Operational experience has shown this control to be effective and robust. This analog controller has been implemented as a digital algorithm in the plasma control system (PCS) [2]. This was a straightforward development of digital filters to match the analog frequency response [3].

\section{Plasma Parameter Estimation}

Off-line estimates of plasma shape and position in DIII-D are calculated using an iterative nonlinear algorithm known as EFIT for fitting equilibria to diagnostic measurements. The EFIT reconstruction code [4] is routinely used at DIII-D and other laboratories world-wide. Plasma reconstruction is based on a current profile and flux distribution consistent with the Grad-Shafranov tokamak equilibrium relation [5]. The effects of variation of plasma pressure, internal inductance, and edge current density on shape identification are handled properly. Certain other diagnostic data can be included to directly determine the spatial distribution of current across the plasma.

Real time shape estimation has traditionally been achieved at DIII-D by a set of approximate formulas derived partly from theory and partly from fits to "data" (EFIT reconstructed equilibria) [6]. These formulas are used to estimate quantities to be controlled such as the vertical position of the plasma current center $\left(Z_{\text {cur }}\right)$, inner plasma to vessel gap $\left(\mathrm{GAP}_{\mathrm{in}}\right)$, plasma top to vessel gap $\left(\mathrm{GAP}_{\text {top }}\right)$, major radius of the center of the outer flux surface $\left(\mathbf{R}_{\text {surf }}\right)$, $\mathrm{X}$-point vertical position $\left(\mathrm{Z}_{\mathrm{X}}\right), \mathrm{X}$-point radial position $\left(\mathrm{RX}_{\mathrm{X}}\right)$ and, indirectly, plasma elongation and triangularity (Fig. 2). The X-point is the location inside the vessel where the poloidal magnetic field $\mathrm{B}$ is equal to 0 . Magnetic field lines correspond to the contours of constant flux shown.

More recently, a modified version of the EFIT algorithm which produces a new equilibrium estimate every $1.5 \mathrm{~ms}$ [7] was implemented to provide more accurate real time estimates of plasma shape and position. The real time algorithm provides shape identification with accuracy previously produced only by between shot analysis. Another advantage is that this single shape identification method handles all discharge shapes for DIII-D experiments. Previously, each distinct plasma shape required calculating a different set of controlled parameters.

\section{Changing Control Paradigms}

For the most part control at DIII-D has been done in the past with PID algorithms which map a single parameter such as one of the plasma to vessel gaps, plasma elongation, triangularity, or X-point location to a single or small number of nearby shaping coils $[6,8]$. Perturbations caused by one of these controls on other plasma parameters were ignored.

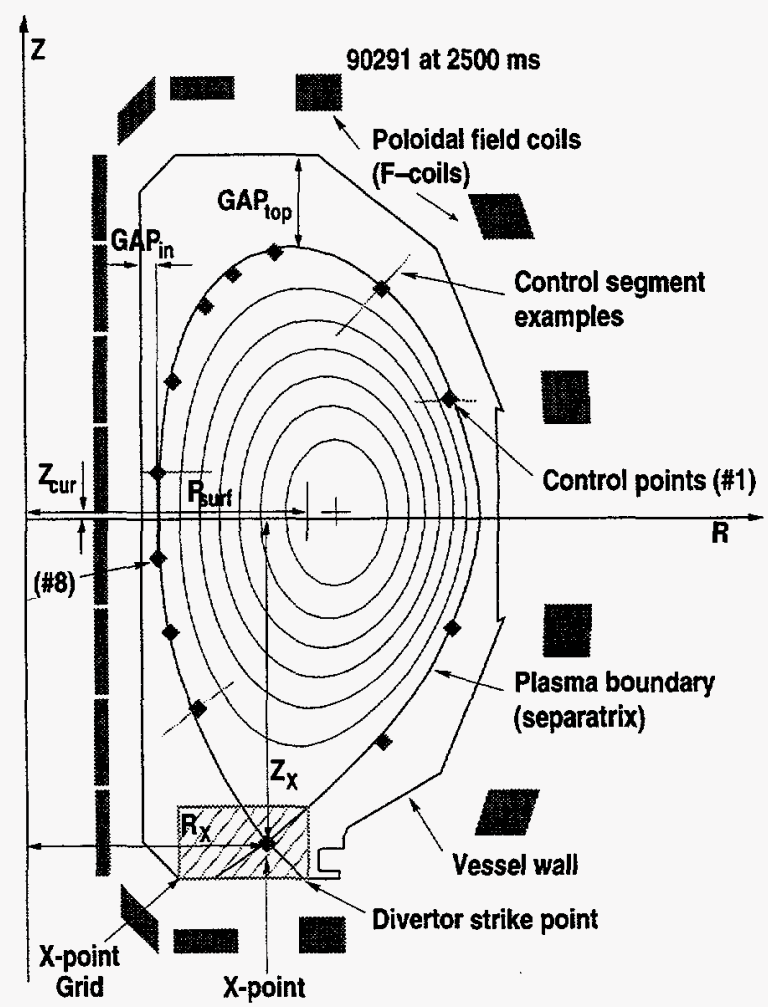

Fig. 2. Examples of controlled plasma parameters prior to realtime EFIT implementation (GAP in, GAP top, $Z_{c u r}$, $\left.R_{\text {surf, }} Z_{x}, R_{x}\right)$ and in new isoflux control $\left(R_{x}, Z_{x}\right.$, and flux at control points \#1-\#13 on control segments). 
Methods for plasma control have evolved in parallel with improvements in the estimation of plasma shape and position. The most recent change of control methodology has been the transition from so-called "gap control" described above to "isoflux" control [6] which exploits the capability of the new real time EFIT algorithm to calculate magnetic flux at specified locations within the tokamak vessel. Figure 2 illustrates a plasma which was controlled using the new isoflux control. Real time EFIT can calculate very accurately the value of flux in the vicinity of the plasma boundary. Thus, the controlled parameters become the values of flux at prespecified control points along with the $X$-point $r$ and $z$ position. By requiring that the flux at each control point be equal to the same constant value, the control forces the same flux contour to pass through all of these control points. By choosing this constant value equal to the flux at the X-point, this flux contour must be the last closed flux surface or separatrix. The desired separatrix location is specified by selecting one of a large number of control points along each of several control segments (Fig. 2). An X-point control grid is used to assist in calculating the $X$-point location by providing detailed flux and field information at a number of closely spaced points in the vicinity of the X-point.

\section{Tokamak Models}

To develop a multivariable controller for poloidal shaping, it is necessary to develop and validate a model of the system(s) to be controlled. Models of these systems include a highly nonlinear plasma, a large but linear set of circuit equations defining shaping currents as a function of applied voltage, and shaping power supplies which also require highly nonlinear models. The modeling of plasma response to external shaping coil currents is an active area of research and will be discussed below. To reduce the complexity of the plant model for control design, individual controllers for the shaping power supplies were developed and implemented [3] to provide an approximately "ideal" voltage source.

5.1. DIII-D Power Systems and Conductors. Figure 3 shows F-coil circuit connections used when producing a typical lower single null (X-point near bottom of vessel) plasma. The boxes labeled $\mathrm{V}$ represent voltage sources (shaping power supplies). Note that these are in series with the F-coils and thus do not control F-coil voltages directly. The E-power supply (EPS) is used to control current in the E-coil and for purposes of shaping control, simply provides a disturbance input. This diagram, taken together with values of resistances and mutual inductances between all coils and from coils to a segmented vessel model [9] is used to construct the circuit model

$$
M_{d d} d I_{d} d t+R_{d} I_{d}=V_{d}
$$

of external toroidal conductors (i.e. those conductors which influence poloidal shape) in DIII-D. Here, $M_{d d}$ and $R_{d}$ are the conductor-to-conductor mutual inductance matrix and the (diagonal) conductor resistance matrix, both modified by circuit loop connections, $I_{d}$ is the vector

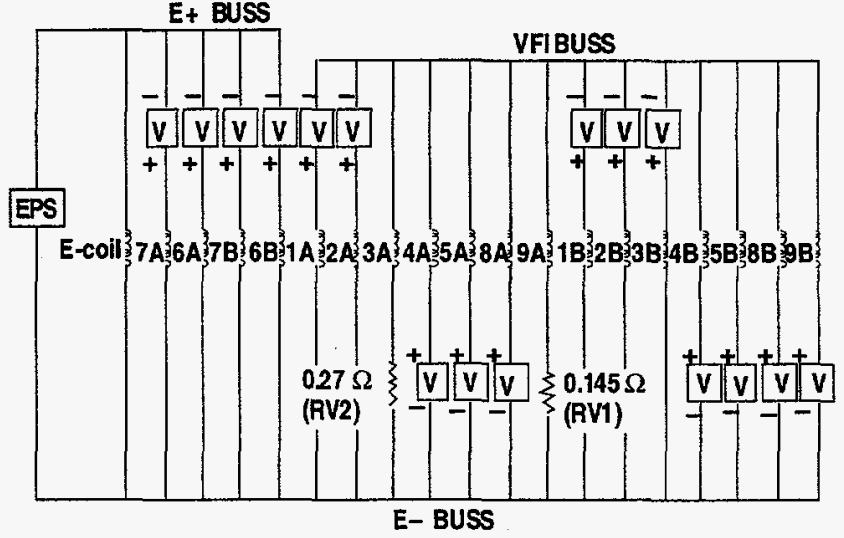

Fig. 3. Model of external shaping coils circuit.

of currents in all conductors, and $V_{d}$ is the vector of voltages in series with the $\mathrm{E}$ - and F-coils. This model, along with models of the various power supplies, has been extensively validated [9].

5.2. Plasma Response Modeling. The plasma response consists of the variations in flux at conductors resulting from plasma shape changes. The plasma shape in turn varies in response to conductor currents. The effect of the plasma therefore can be modeled as an additional mutual inductance term in the conductor circuit equation, which becomes

$$
M_{d d} \dot{I}_{d}+R_{d} I_{d}+X_{d d} \dot{I}_{d}=V_{d}
$$

In this equation, $X_{d d}$ describes flux variations at conductors due to plasma shape and position changes which in turn arise from conductor current changes.

The response of the plasma to coil currents $\left(X_{d d}\right)$ can contribute variations in flux at conductors comparable to flux variations from conductor currents alone $\left(M_{d d}\right)$. In particular, variations in conductor flux resulting from vertical motion of the plasma are dominated by the plasma contribution, giving rise to the only unstable mode of the system, known as the "vertical instability". Use of a plasma response model is thus necessary at some level to produce an efficient stabilizing controller, and can in principle improve control of the remaining stable modes.

Two general approaches are available to determine the lin earized plasma response for DIII-D: system identification through experimentation or computational simulation, and direct determination of the linear response through perturbational or energy principle methods. We have decided against the first approach because generation of models for the wide range of DIII-D plasmas would be extremely expensive and reliance on such an approach would make it difficult to generate new plasma equilibria.

The second approach involves explicit perturbation of conductor currents in equilibrium calculations to determine the linearized response of the plasma [10]. Alternatively, trial function stationization of the system 
Lagrangian, derived from the ideal MHD energy principle, provides a description of a perfectly flux-conserving plasma. However, both of these require knowledge and specification of certain constraints imposed by the physical plasma. A (idealized) plasma which obeys ideal MHD is constrained to conserve flux at all points within the plasma. Physical plasmas, by contrast, tend to be increasingly resistive near the plasma surface. Sufficiently high resistivity of the edge plasma region can dissipate flux-conserving induced currents on time scales of interest in plasma shaping control, causing the plasma response to be quite different from ideal MHD.

For example, experimental observations suggest that some characteristics of the X-point and strike point (Fig. 2) response can be highly sensitive to the choice of flux conservation constraint. Figure 4 illustrates the effect of downward motion of the plasma on the field line geometry around the X-point in DWI-D. The solid and dashed contours show the separatrix at two different times. The absence of significant edge current density (equivalently, lack of flux conservation) is indicated by the preservation of right angles in the X-point field pattern during the evolution. Plasmas with better confinement and therefore possibly higher edge temperatures are routinely produced in DIII- D, and may conserve edge flux sufficiently well to dramatically change the $\mathrm{X}$-point response. Because the uncertainty in the temperature (and hence resistivity) of the edge plasma can be very great, such sensitivity to modeling assumptions may pose problems for plasma response models on which control action is made to depend.

Unfortunately, the best choice of flux constraint to accurately characterize physical plasma shape response in general is not yet clear. Although many detailed comparisons of resistive MHD simulation codes with experimental plasma shape responses have been performed in recent years using such axisymmetric resistive $\mathrm{MHD}$ simulation codes as TSC [11] and DINA [12], the implications of these results for linear plasma response modeling have not been completely assessed.

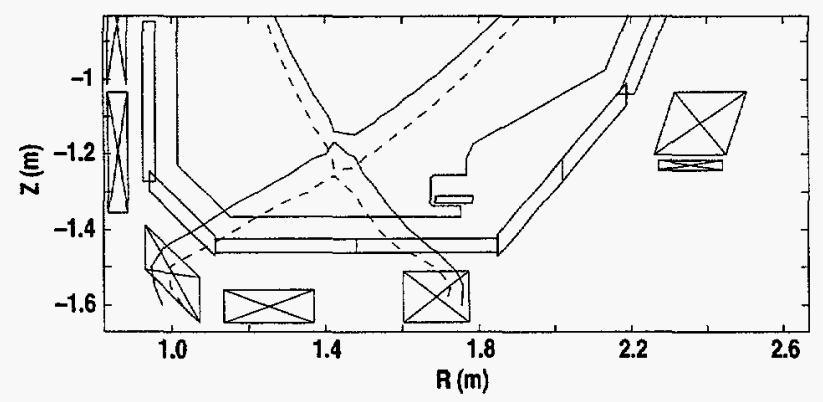

Fig. 4. Evolution of X-point and magnetic field line geometry during plasma vertical motion. The angles forming the $X$-point continue to be right angles throughout the evolution, indicating negligible edge current density.
Despite the ambiguity in choices of plasma edge conductivity, both nonlinear simulations and linear models derived therefrom can reproduce experimental vertical instability growth rates and the trajectory of the plasma center [13]. Growth rates and linear trajectories typically agree to better than $10 \%$ when detailed conductor and power supply models are used in such simulations and models [14]. The linear response of the plasma-wall gap spacings to conductor variation derived from perturbed conductor currents has been shown to agree approximately (typically within 10\%-50\%) with plasma nonlinear code responses [15]. General shape control may therefore be satisfactorily accomplished using detailed plasma response models for the control design. However, more detailed control such as regulation of strike point locations may require the ability to accurately predict the edge conductivity conditions.

\section{Multivariable Control Design}

Current work which seeks to exploit the new real time equilibrium reconstruction capability and the "isoflux" control approach is the development of true multivariable controllers which can account for the many dependencies of flux at various locations on currents in shaping coils.

As described above, in DIII-D the behavior of the X-point geometry may vary significantly from one kind of discharge to another. Thus, fine control of the X-point and nearby magnetic field lines may require specific controllers for each kind of discharge, or even gain scheduling of controllers during a single discharge. If such high performance control is not required, controllers designed without reference to a specific plasma model may be sufficient. The only control which clearly requires a model for development is the vertical position control and this uses a separate algorithm. In fact, previous shape controller designs on DIII-D and on other devices have essentially ignored the effect of the plasma and controlled plasmas "reasonably well" $[6,14,16]$. Others have used some form of plasma model in their designs, also with success $[17,18]$.

Our initial work on this problem attempts to understand through simulations the impact of the choice of models on development and implementation of controllers for DIII-D. Initially we tend to favor reduced complexity over fine-scale control. The least complex option would be if we were able to design controllers without reference to a plasma model. Failing that, cruder models which were appropriate over many different plasmas would be preferred. The first plasma model used in our simulations is a crude one degree of freedom approximation in which plasma current is concentrated in a single filament at the plasma current centroid and only rigid radial plasma motion is allowed.

We chose to use the normalized coprime factorization (NCF) [19] design technique to design controllers and to test them in a closed loop mixed linear/nonlinear plasma 
simulation, shown in Fig. 5. It uses a linearized plant to evolve coil and vessel currents, followed by a nonlinear algorithm which performs the following computations: (1) calculation of $B_{r}$ and $B_{Z}$ at the desired $X$-point location (target), (2) estimate X-point location and flux at X-point using the same algorithm as in the real time code, (3) compute flux at specified control points. Control of the X-point location in DIII-D experiments using the isoflux technique has been done by feeding back the $\mathrm{X}$-point $\mathrm{r}$ and $\mathrm{z}$ errors directly to a controller. The simulation on the other hand attempts to control its location by forcing $\mathrm{B}_{\mathrm{r}}=\mathrm{B}_{\mathrm{Z}}=0$ at the targeted $\mathrm{X}$-point location. The flux errors (flux at control points minus calculated $X$-point flux), $\mathrm{B}_{\mathrm{r}}$ and $\mathrm{B}_{\mathrm{Z}}$ at the target $\mathrm{X}$-point, and the $\mathrm{F}$-coil currents are fed back to the controller which calculates a voltage demand for the shape control power supplies. We assume here that the control of the power supplies is good enough that this voltage can be instantaneously achieved relative to the timescale needed for effective shaping control (order of $1 \mathrm{~ms}$ ). These supplies have a (soft) limited voltage range $( \pm 250$ to $\pm 350 \mathrm{~V})$ so voltages are clipped in simulation.

Plasma shape controllers were designed with a plant model which assumed that the flux/field at all points on a control segment (and over the X-point grid) in response to a given conductor current is equal to the average over the segment (grid) of the true responses to that current. This appears to be a reasonable approximation for flux responses, since flux always increases with increasing current, i.e. the sign of the response remains constant. However, for certain F-coils, the direction of change in field at a grid point with increasing current depends on which point on the grid is being measured. The effect of this difference in sign is that the same change in current in coil F8B, for example, will sometimes push the X-point to the right and sometimes to the left, depending on where the $\mathrm{X}$-point is initially.

Several controllers were designed and tested in simulation. Those controllers which were designed with knowledge of plasma model all stabilized very well and traded off such quantities as response time, voltages used, coil current excursions, and stability robustness as measured by the NCF robustness parameter $\varepsilon$. An example is shown in Fig. 6 . Note that $X$-point control has been emphasized at

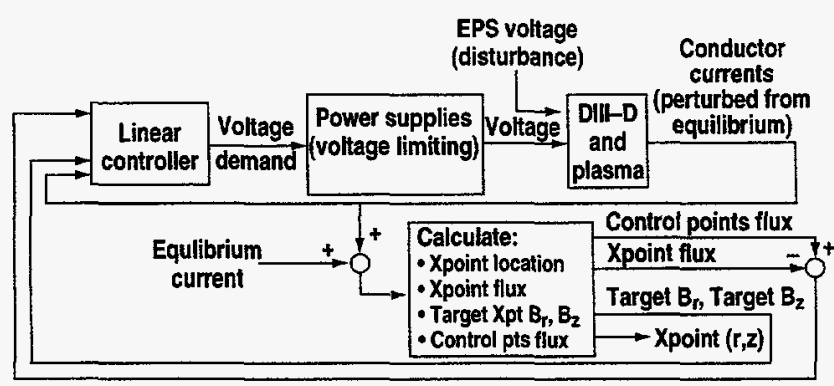

Fig 5. Closed loop simulation of DIII-D, including plasma, with multivariable controller. the expense of boundary control because of the impact in DIII-D of the strike point location on the ability to perform certain physics studies. Also note that prompt response of $B_{r}$ and $B_{z}$ errors does not translate to equally prompt response of $\mathrm{X}$-point $\mathrm{r}$ and $\mathrm{z}$ errors, lending support to a previous decision to directly feed back the $X$-point $r$ and $\mathrm{z}$ locations rather than control the $\mathrm{X}$-point location through control of the magnetic field.

While voltages remain well below their limits in Fig. 6 we see that coil currents are growing large. In general, current limits (a few kAmps per coil) are easily reached with an inappropriate choice of target boundary location which is incompatible with reasonable equilibrium coil currents. This issue of definition of target shapes compatible with limited coil currents is a problem which must also be addressed in order for a multivariable controller to become operational.

Several unsuccessful attempts were also made to design a controller using only the conductors model without the

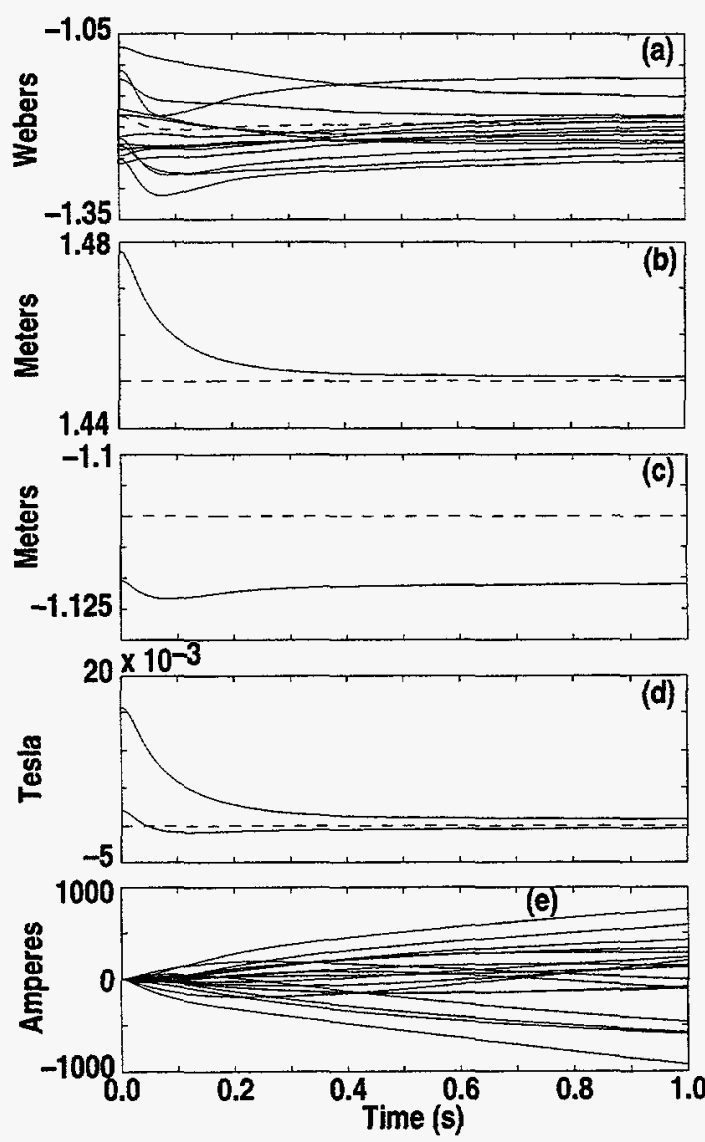

Fig. 6. Example of closed loop response with controller designed having knowledge of plasma response: (a) measured flux on segment control points (dashed line = flux at $X$-point), (b) X-point $r$ coordinate and desired value, (c) X-point $z$ coordinate and desired value, (d) $B_{r}$ and $B_{z}$ at target $X$-point location, (e) F-coil current perturbations from equilibrium. 
plasma response which would provide "reasonable" control of the crude plasma we are using. All such controllers were very robustly closed loop stable (as measured by $\varepsilon$ ) for the plasmaless system, but all destabilized the initially stable plasma-included plant in simulation.

It's possible that alternative design techniques which include modeling of system uncertainties (e.g. [20]) could be used to design a workable controller using a plasmaless model. In this case, we can include models for bounds on perturbations of the nominal (no plasma) system which would include the physical system. To illustrate the perfurbation size, in our example models describing the response $y$ of flux and field at control points are given by state space descriptions $d_{I} / d t=A_{I}+B V, y=C_{I}+D_{V}$, with

Aplasma $=$ Anopl $+\Delta \mathrm{A},\|\Delta \mathrm{A}\| / \|$ Anopl $\|=0.0399$

Bplasma $=\mathrm{Bnopl}+\Delta \mathrm{B}, \quad\|\Delta \mathrm{B}\| / / \mathrm{BBnop} \|=0.0025$

Cplasma $=\mathrm{Cnopl}+\Delta \mathrm{C}, \quad\|\Delta \mathrm{C}\| /\|\mathrm{Cnop}\|=1.3985$

Dplasma $=$ Dnopl $=0$,

where the "plasma" subscript indicates that the system includes plasma effects, "nopl" indicates it does not. Note that the dynamics of the current evolution in the external conductors will likely not be much different with or without a plasma present. The flux and field response to conductor currents is significantly different however.

\section{Conclusion}

In this paper we have discussed the techniques used now and in the past to control plasma shape and position of the DIII-D tokamak plasmas. Emphasis has been placed on the ongoing transition to more sophisticated control methods which require models of the system to be controlled in order to develop controllers. Much has been learned in the fusion community about the adequacy of plasma models for predicting plasma evolution, but the issue of how sophisticated a model is really necessary for effective multivariable controller design is not yet resolved. We believe that some information about the effect of the plasma is needed. Our conjecture is that a model only slightly more sophisticated than the rigid radial response plasma we use here is necessary, except perhaps for detailed control of field lines around the $X$-point. We noted that, around the $\mathrm{X}$-point, a change in model assumption (flux conserving or flux dissipating) can dramatically alter the assumed motion of the field lines in response to plasma motion and thus to external conductor current changes.

With further work, we hope to verify the above conjecture and to investigate whether there do in fact exist physical plasmas which have dynamically driven edge currents significant enough to affect control response. Once these issues are resolved, we need to produce a small collection of (simple) models which will allow design of effective multivariable controllers, and then implement these controllers on DIII-D.

\section{References}

[1] E.A. Lazarus, J.B. Lister, G.H. Nielson, "Control of the vertical instability in tokamaks," Nucl. Fusion 30, 111 (1990).

[2] J.R. Ferron, et al., "An advanced plasma control system for the DIII-D tokamak," in Proc. 14th IEEE/NPSS Symp. on Fusion Engineering, p. 761.

[3] M.L.Walker, et al., "Status of DIII-D plasma control," in Proc. 16th IEEE/NPSS Symp. on Fusion Engineering, 1995, p. 885.

[4] L.L. Lao, et al., Nucl. Fusion 25, 1611 (1985).

[5] J. Wesson, Tokamaks, Clarendon Press, Oxford, 1987.

[6] S. Kinoshita, et al., "Independent control of gaps in single-null divertor discharges on the DIII-D tokamak," General Atomics Report GA-A19584 (1989).

[7] J.R. Ferron, et al., "Real time equilibrium reconstruction for tokamak discharge control," General Atomics Report GA-A22586, (1997), submitted to Nucl. Fusion.

[8] J.S. Haskovec, et al., "Analog computation system for plasma position and control," General Atomics Report GA-A18972 (1987)

[9] M.L. Walker, A. Nerem, D. Baggest, "Advanced plasma control final report, part I: DIII-D system models for feedback control," General Atomics Report GAC21745 (1996).

[10] R. Albanese, et al., "Plasma modeling for vertical instabilities," Nucl. Fusion 29, 1013 (1989).

[11] S.C. Jardin, N. Pomphrey, J. De Lucia, J. Comput. Phys. 50, 481 (1986).

[12] R.R. Khayrutdinov, V.E. Lukash, "Studies of plasma equilibrium and transport in a tokamak fusion device with the inverse-variable technique," J. Comput. Phys 109, 193 (1993).

[13] R.O. Sayer, et al., Nucl. Fusion 33, 969 (1993).

[14] J.B. Lister, et al., "The control of TCV plasmas," to be published in Fusion Technol.

[15] J.B. Lister, et al., "The ITER plasma response in linear and nonlinear models," submitted to Nucl. Fusion.

[16] I.H. Hutchinson, et al., "Plasma shape control: a general approach and its application to Alcator C-Mod," Fusion Technol. 30, 137 (1996).

[17] M. Kikuchi, H. Ninomiya, R. Yoshino, S. Seki, “A matrix transfer function analysis applied to the active feedback control system of a divertor tokamak," Nucl. Fusion 27, 299 (1987).

[18] M. Garribba, R. Litunovshky, and S. Puppin, "The plasma shape and control system for JET," Fusion Technol. 31 (1997).

[19] D.C. McFarlane, K. Glover, "Robust controller design using normalised coprime factor plant descriptions," Springer-Verlag, Lect. Notes in Control and Inf. Sci., v.ol 138, 1989

[20] J.C. Doyle, K. Glover, P. Khargonekar, B. Francis, "State-space solutions to standard $\mathrm{H}_{2}$ and $\mathrm{H}$-infinity control problems," IEEE Trans. Aut. Cont. 34, (1989). 